\title{
Uniform distribution of primes having a prescribed primitive root
}

\author{
by \\ Pieter Moree (Bonn and Amsterdam)
}

1. Introduction. If $S$ is any set of prime numbers, denote by $S(x)$ the number of primes in $S$ not exceeding $x$. For given integers $a$ and $d$, denote by $S(x ; a, d)$ the number of primes in $S$ not exceeding $x$ that are congruent to $a$ modulo $d$. We say that $S$ is weakly uniformly distributed mod $d$ if $S$ is infinite and for every $a$ coprime to $d$,

$$
S(x ; a, d) \sim \frac{S(x)}{\varphi(d)},
$$

where $\varphi(d)$ denotes Euler's totient function. In case $S$ is infinite the progressions $a(\bmod d)$ such that the latter asymptotic equivalence holds are said to get their fair share of primes from $S$. Thus $S$ is weakly uniformly distributed mod $d$ if and only if all the progressions mod $d$ get their fair share of primes from $S$. W. Narkiewicz [7] has written a nice survey on the state of knowledge regarding the (weak) uniform distribution of many important arithmetical sequences.

In this paper the weak uniform distribution of a class of sequences, apparently not considered in this light before, will be investigated. Let $G$ be the set of non-zero rational numbers $g$ such that $g \neq-1$ and $g$ is not a square of a rational number. Let $\mathcal{P}_{g}$ denote the set of primes $p$ such that $g$ is a primitive root modulo $p$. Clearly a necessary condition for $\mathcal{P}_{g}$ to be infinite is that $g \in G$. That this is also a sufficient condition was conjectured by Emil Artin in 1927 and is called Artin's primitive root conjecture. There is no value of $g$ for which $\mathcal{P}_{g}$ is known to be infinite. Presently the best unconditional result on Artin's conjecture is due to R. Heath-Brown [1]. Heath-Brown's result implies that there are at most two primes $q$ for which $\mathcal{P}_{q}$ is finite. Assuming GRH, C. Hooley [2] proved in 1967 a quantitative version of Artin's conjecture (Theorem 4 below with $f=1$ and $g \in G \cap \mathbb{Z}$ ). In this note we will make use of the following straightforward generalization

1991 Mathematics Subject Classification: 11R45, 11A07, 11N69. 
of Hooley's result. As usual, $\mu$ and $\zeta_{n}$ denote the Möbius function and a primitive root of unity of order $n$, respectively.

Theorem 1 [4]. Let $M$ be Galois and $g \in G$. Suppose the Riemann Hypothesis holds for the fields $M\left(\zeta_{k}, g^{1 / k}\right)$ for every squarefree $k$. Then $N_{M}(g ; x)$, the number of primes $p$ not exceeding $x$ that split completely in $M$ and such that $g$ is a primitive root $\bmod p$, satisfies

$$
N_{M}(g ; x)=\left(\sum_{k=1}^{\infty} \frac{\mu(k)}{\left[M\left(\zeta_{k}, g^{1 / k}\right): \mathbb{Q}\right]}\right) \frac{x}{\log x}+O\left(\frac{x \log \log x}{\log ^{2} x}\right) .
$$

For $g \neq-1,0,1$ define

$$
\delta(M, g):=\sum_{k=1}^{\infty} \frac{\mu(k)}{\left[M\left(\zeta_{k}, g^{1 / k}\right): \mathbb{Q}\right]} .
$$

(Since $\left[M\left(\zeta_{k}, g^{1 / k}\right): \mathbb{Q}\right] \gg k \varphi(k)$, the series is seen to converge, even absolutely, and hence $\delta(M, g)$ is well defined.) Hooley computed $\delta(\mathbb{Q}, g)$ for $g \in G \cap \mathbb{Z}$. It turns out that $\delta(\mathbb{Q}, g) \neq 0$ for such $g$ and thus Artin's conjecture holds true, on GRH. In particular $\delta(\mathbb{Q}, g)$ is a rational number times

$$
A=\prod_{p}\left(1-\frac{1}{p(p-1)}\right) \quad(\approx .3739558)
$$

the so-called Artin constant. For example, taking $f=1, g=2$ and $M=\mathbb{Q}$ in Theorem 4 yields $\mathcal{P}_{2}(x) \sim A x / \log x$. In this paper $\delta(M, g)$ will be computed for $M$ cyclotomic (Theorem 4). This result is then used to compute, on GRH, the set $D_{g}$ of natural numbers $d \geq 1$ such that $\mathcal{P}_{g}$ is weakly uniformly distributed mod $d$. In Theorem 2 simple sets $S_{g}$ are indicated such that $D_{g}=S_{g}$. Theorem 4 allows one to prove that $D_{g} \subseteq S_{g}$. The work of H. Lenstra [4] is used to prove that $D_{g} \supseteq S_{g}$.

In [9] F. Rodier, in connection with a coding-theoretical result involving Dickson polynomials, made the conjecture that

$$
\mathcal{P}_{2}(x ; 3,28)+\mathcal{P}_{2}(x ; 19,28)+\mathcal{P}_{2}(x ; 27,28) \sim \frac{A}{4} \cdot \frac{x}{\log x} .
$$

Note that weak uniform distribution $\bmod 28$ of $\mathcal{P}_{2}$ would imply Rodier's conjecture. In [6] it was shown that, on GRH, $D_{2}=\{1,2,4\}$, and thus $\mathcal{P}_{2}$ is not weakly uniformly distributed $\bmod 28$. Moreover, it was shown, on $\mathrm{GRH}$, that the true constant in (2) is $21 A / 82$. Another coding-theoretical application of primitive roots in arithmetic progressions occurs in the theory of perfect arithmetic codes [5].

In Theorem $2, D_{g}$ is computed for $g \in G$. Notice that we can uniquely write $g=g_{1} g_{2}^{2}$, with $g_{1}$ a squarefree integer and $g_{2} \in \mathbb{Q}_{>0}$. Let $h$ be the largest integer such that $g$ is an $h$ th power. Notice that $g \in G$ implies that $h$ must be odd. 
TheOREM $2(\mathrm{GRH})$. Let $g \in G$, and let $h$ be the largest integer such that $g$ is an hth power. Assume that either $g_{1} \neq 21$ or $(h, 21) \neq 7$. Then $D_{g}$, the set of natural numbers $d$ such that the set of primes $p$ such that $g$ is a primitive root mod $p$ is weakly uniformly distributed mod $d$, equals

(i) $\left\{2^{n}: n \geq 0\right\}$ if $g_{1} \equiv 1(\bmod 4)$;

(ii) $\{1,2,4\}$ if $g_{1} \equiv 2(\bmod 4)$;

(iii) $\{1,2\}$ if $g_{1} \equiv 3(\bmod 4)$.

In the remaining case $g_{1}=21$ and $(h, 21)=7$, we have $D_{g}=\left\{2^{n} 3^{m}\right.$ : $n, m \geq 0\}$.

For simplicity we call $g$ exceptional if $g_{1}=21$ and $(h, 21)=7$ and ordinary otherwise. The following variant of Theorem 2 sheds some light on (i), (ii) and (iii) of Theorem 2 :

TheOrem $3(\mathrm{GRH})$. Let $g$ and $h$ be as in Theorem 2 and assume that $g$ is ordinary. Then $\mathcal{P}_{g}$ is weakly uniformly distributed modulo $d$ if and only if for every squarefree $k \geq 1, \mathbb{Q}\left(\zeta_{k}, g^{1 / k}\right) \cap \mathbb{Q}\left(\zeta_{d}\right)=\mathbb{Q}$.

Let $g$ be exceptional and $d$ be of the form $2^{\alpha} 3^{\beta}$ with $\beta \geq 1$. It turns out, on GRH, that $\mathcal{P}_{g}$ is weakly uniformly distributed mod $d$. On the other hand, there exist $k$ such that $\mathbb{Q}\left(\zeta_{k}, g^{1 / k}\right) \cap \mathbb{Q}\left(\zeta_{d}\right)=\mathbb{Q}(\sqrt{-3})$ (cf. the remark following Lemma 7). Thus the requirement " $g$ is ordinary" in Theorem 3 cannot be dropped.

2. The density of primes $p \equiv 1(\bmod f)$ having a prescribed primitive root. In this section Theorem 4 will be proved. This result gives, on GRH, for arbitrary $f \geq 1$ the density of primes $p$ such that $p \equiv 1(\bmod f)$ and moreover a prescribed integer $g$ is a primitive root $\bmod p$. Theorem 1 relates this density to the degrees of the fields $M\left(\zeta_{k}, g^{1 / k}\right)$ with $M$ cyclotomic (namely $M=\mathbb{Q}\left(\zeta_{f}\right)$ ). These degrees are computed in Lemma 2, making use of the following well known fact from cyclotomy (see e.g. [10, p. 163]).

Lemma 1. Let $0 \neq a \in \mathbb{Q}$. Write $a=a_{1} a_{2}^{2}$, with $a_{1}$ a squarefree integer and $a_{2} \in \mathbb{Q}$. Then the smallest cyclotomic field containing $\mathbb{Q}(\sqrt{a})$ is $\mathbb{Q}\left(\zeta_{\left|a_{1}\right|}\right)$ if $a_{1} \equiv 1(\bmod 4)$ and $\mathbb{Q}\left(\zeta_{4\left|a_{1}\right|}\right)$ otherwise.

Lemma 1 can also be phrased as: the smallest cyclotomic field containing $\mathbb{Q}(\sqrt{a})$ is $\mathbb{Q}\left(\zeta_{\left|\Delta_{a}\right|}\right)$, with $\Delta_{a}$ the discriminant of $\mathbb{Q}(\sqrt{a})$.

The next result can be proved by a trivial generalization of an argument given by Hooley [2, pp. 213-214].

LEMma 2. Let $g \in G$, and let $h$ be the largest positive integer such that $g$ is an hth power. Let $\Delta$ denote the discriminant of $\mathbb{Q}(\sqrt{g})$. Suppose that $k \mid r$ and $k$ is squarefree. Put $k_{1}=k /(k, h)$ and $n(k, r)=\left[\mathbb{Q}\left(\zeta_{r}, g^{1 / k}\right): \mathbb{Q}\right]$. Then 
(i) for $k$ odd, $n(k, r)=k_{1} \varphi(r)$;

(ii) for $k$ even and $\Delta \nmid r, n(k, r)=k_{1} \varphi(r)$;

(iii) for $k$ even and $\Delta \mid r, n(k, r)=k_{1} \varphi(r) / 2$.

Proposition 1. Let $f, h \geq 1$ be integers. Then the function $w: \mathbb{N} \rightarrow \mathbb{N}$ defined by

$$
w(k)=\frac{k \varphi(\operatorname{lcm}(k, f))}{(k, h) \varphi(f)}
$$

is multiplicative.

Proof. For every multiplicative function $g$ and arbitrary integers $a, b \geq 1$, we obviously have $g(a) g(b)=g(\operatorname{gcd}(a, b)) g(\operatorname{lcm}(a, b))$. Hence, to finish the proof it is enough to show that $\varphi((k, f))$ is a multiplicative function of $k$, which is obvious.

TheOREM 4. Let $g \in G$, and let $h$ be the largest integer such that $g$ is an hth power. Let $f \geq 1$ be an arbitrary integer. Let $\Delta$ denote the discriminant of $\mathbb{Q}(\sqrt{g})$. Put $b=\Delta /(\Delta, f)$. Let $w(k)$ be as in Proposition 1. Put

$$
A(f, h)=\prod_{\substack{p \nmid f \\ p \mid h}}\left(1-\frac{1}{p-1}\right) \prod_{\substack{p \mid f \\ p \nmid h}}\left(1-\frac{1}{p}\right) \prod_{\substack{p \nmid f \\ p \nmid h}}\left(1-\frac{1}{p(p-1)}\right) .
$$

Let $N_{\mathbb{Q}\left(\zeta_{f}\right)}(g ; x)$ denote the number of primes $p$ not exceeding $x$ that split completely in $\mathbb{Q}\left(\zeta_{f}\right)$ and such that $g$ is a primitive root mod $p$. If $(f, h)>1$, then $\delta\left(\mathbb{Q}\left(\zeta_{f}\right), g\right)=0$ and $N_{\mathbb{Q}\left(\zeta_{f}\right)}(g ; x)$ is bounded above.

Next assume that $(f, h)=1$. Then

$$
\begin{aligned}
\delta\left(\mathbb{Q}\left(\zeta_{f}\right), g\right) & =\frac{1}{\varphi(f)}\left(1-\frac{\mu(|b|)}{\prod_{p \mid b}(w(p)-1)}\right) \prod_{p}\left(1-\frac{1}{w(p)}\right) \\
& =\frac{A(f, h)}{\varphi(f)}\left(1-\frac{\mu(|b|)}{\prod_{p|b, p| h}(p-2) \prod_{p \mid b, p \nmid h}\left(p^{2}-p-1\right)}\right)
\end{aligned}
$$

if either $g_{1} \equiv 1(\bmod 4)$, or $g_{1} \equiv 2(\bmod 4)$ and $8 \mid f$, or $g_{1} \equiv 3(\bmod 4)$ and $4 \mid f$. Otherwise

$$
\delta\left(\mathbb{Q}\left(\zeta_{f}\right), g\right)=\frac{1}{\varphi(f)} \prod_{p}\left(1-\frac{1}{w(p)}\right)=\frac{A(f, h)}{\varphi(f)} .
$$

Suppose the Riemann Hypothesis holds for the field $\mathbb{Q}\left(\zeta_{f}, \zeta_{k}, g^{1 / k}\right)$ for every squarefree $k$. Then

$$
N_{\mathbb{Q}\left(\zeta_{f}\right)}(g ; x)=\delta\left(\mathbb{Q}\left(\zeta_{f}\right), g\right) \frac{x}{\log x}+O\left(\frac{x \log \log x}{\log ^{2} x}\right) .
$$


Proof. We have to evaluate

$$
\delta\left(\mathbb{Q}\left(\zeta_{f}\right), g\right)=\sum_{k=1}^{\infty} \frac{\mu(k)}{\left[\mathbb{Q}\left(\zeta_{\mathrm{lcm}(k, f)}, g^{1 / k}\right): \mathbb{Q}\right]} .
$$

From Lemma 2 it follows that

$$
\begin{aligned}
\varphi(f) \delta\left(\mathbb{Q}\left(\zeta_{f}\right), g\right) & =\sum_{\substack{k=1 \\
2 \nmid k}}^{\infty} \frac{\mu(k)}{w(k)}+\sum_{\substack{k=1 \\
\Delta \nmid \operatorname{lcm}(2 k, f)}}^{\infty} \frac{\mu(2 k)}{w(2 k)}+2 \sum_{\substack{k=1 \\
\Delta \mid \operatorname{lcm}(2 k, f)}}^{\infty} \frac{\mu(2 k)}{w(2 k)} \\
& =\sum_{k=1}^{\infty} \frac{\mu(k)}{w(k)}+\sum_{\substack{k=1 \\
\Delta \mid \operatorname{lcm}(2 k, f)}}^{\infty} \frac{\mu(2 k)}{w(2 k)}=I_{1}+I_{2} .
\end{aligned}
$$

I claim that

$$
I_{1}=\prod_{p}\left(1-\frac{1}{w(p)}\right) \quad \text { and } \quad I_{2}=\frac{\mu(2|b|)}{w(|b|)} \prod_{p \nmid b}\left(1-\frac{1}{w(p)}\right) .
$$

Indeed, the arithmetic function $w$ is multiplicative by Proposition 1 and thus, by Euler's identity, $I_{1}=\prod_{p}(1-1 / w(p))$. Further, if $b$ is even, then $I_{2}=\mu(2|b|)=0$. Next assume that $b$ is odd. Now $\Delta \mid \operatorname{lcm}(2 k, f)$ is equivalent to $b \mid 2 k /(2 k, f)$. Since $(b,(2 k, f))=1$ and $b$ is odd, $b \mid 2 k /(2 k, f)$ is equivalent to $b \mid k$. Thus

(6) $\quad I_{2}=\sum_{\substack{k=1 \\ b \mid k}}^{\infty} \frac{\mu(2 k)}{w(2 k)}=\frac{\mu(2|b|)}{w(2|b|)} \sum_{\substack{k=1 \\(k, 2 b)=1}}^{\infty} \frac{\mu(k)}{w(k)}=\frac{\mu(2|b|)}{w(2|b|)} \prod_{p \nmid 2 b}\left(1-\frac{1}{w(p)}\right)$.

Using the fact that $b$ is odd and $w(2)=2$ completes the proof of (5).

Using (5) the proof is now easily completed. We distinguish two subcases: $(f, h)>1$ and $(f, h)=1$.

(i) $(f, h)>1$. Since $g \in G, h$ is odd. Since $(b, f) \mid 2$ and $h$ is odd, there is an odd prime $p_{1}$ such that $p_{1}\left|h, p_{1}\right| f$ and $p_{1} \nmid b$. Since $w\left(p_{1}\right)=1$, it follows that $I_{1}=I_{2}=0$ and thus $\delta\left(\mathbb{Q}\left(\zeta_{f}\right), g\right)=0$. Let $p$ be a prime with $p \equiv 1(\bmod f)$ and $p \nmid g$. Then the order of $g \bmod p$ is bounded above by $(p-1) / q_{1}$, where $q_{1}$ is the smallest prime dividing $(f, h)$. Hence $N_{\mathbb{Q}\left(\zeta_{f}\right)}(g ; x)$ is bounded above.

(ii) $(f, h)=1$. Then $w(p)>1$ for every prime $p$. Adding the product expansions in (5) results, on using the fact that $w(p)>1$, in

$$
\delta\left(\mathbb{Q}\left(\zeta_{f}\right), g\right)=\frac{1}{\varphi(f)}\left(1+\frac{\mu(2|b|)}{\prod_{p \mid b}(w(p)-1)}\right) \prod_{p}\left(1-\frac{1}{w(p)}\right) .
$$


Notice that $\prod_{p}(1-1 / w(p))=A(f, h)$ and that

$$
\prod_{p \mid b}(w(p)-1)=\prod_{p|b, p| f}(p-1) \prod_{p|b, p \nmid f, p| h}(p-2) \prod_{p \mid b, p \nmid f, p \nmid h}\left(p^{2}-p-1\right) .
$$

Since $(b, f) \mid 2$, the latter identity simplifies to

$$
\prod_{p \mid b}(w(p)-1)=\prod_{p|b, p| h}(p-2) \prod_{p \mid b, p \nmid h}\left(p^{2}-p-1\right) .
$$

Inserting this in (7) we find

$$
\delta\left(\mathbb{Q}\left(\zeta_{f}\right), g\right)=\frac{A(f, h)}{\varphi(f)}\left(1+\frac{\mu(2|b|)}{\prod_{p|b, p| h}(p-2) \prod_{p \mid b, p \nmid h}\left(p^{2}-p-1\right)}\right) .
$$

On invoking Theorem 1, the proof is easily completed.

Let $g \in G$. From [4, Theorem 8.3] it follows that, under GRH, $\delta\left(\mathbb{Q}\left(\zeta_{f}\right), g\right)$ $=0$ if and only if either $(f, h)>1$ or $\Delta \mid f$. Notice that this is an easy consequence of Theorem 4. Assume GRH and, moreover, $(f, h)=1$. Then the above fact can be reformulated, with the help of Lemma 1 , as $\delta\left(\mathbb{Q}\left(\zeta_{f}\right), g\right)=0$ if and only if $\sqrt{g} \in \mathbb{Q}\left(\zeta_{f}\right)$. This is a particular case of the following result:

TheOREM 5 (GRH). Let $g \in G$, and let $h$ be the largest integer such that $g$ is an hth power. Let $M$ be an abelian number field of conductor $f$. Let $N_{M}(g)$ denote the set of primes $p \in \mathcal{P}_{g}$ such that $p$ splits completely in $M$. Suppose that $(f, h)=1$. Then $\delta(M, g)=0$ if and only if $\sqrt{g} \in M$. Moreover, if $N_{M}(g)$ is infinite, then $\delta(M, g)>0$.

We will deduce Theorem 5 from a result of Lenstra [4, Theorem 4.6], which in this context simplifies to:

Theorem 6. Let $g \in G$ and $M: \mathbb{Q}$ be Galois. Let $\pi=\prod_{l \mid h, l \text { prime }} l$, where $h$ is the largest integer such that $g$ is an hth power. Then if $N_{M}(g)$ is infinite, there exists $\sigma \in \operatorname{Gal}\left(M\left(\zeta_{\pi}\right) / \mathbb{Q}\right)$ with $\left(\left.\sigma\right|_{M}\right)=\operatorname{id}_{M}$ and, for every prime $l$ such that $\mathbb{Q}\left(\zeta_{l}, g^{1 / l}\right) \subseteq M\left(\zeta_{\pi}\right),\left(\left.\sigma\right|_{\mathbb{Q}\left(\zeta_{l}, g^{1 / l}\right)}\right) \neq \operatorname{id}_{\mathbb{Q}\left(\zeta_{l}, g^{1 / l}\right)}$. Conversely, if such a $\sigma$ exists and $G R H$ is true, then $N_{M}(g)$ is infinite and $\delta(M, g)>0$.

In addition we will make use of:

Lemma 3. Let $\mathbb{Q} \nsubseteq \mathbb{Q}(\sqrt{d}) \subseteq \mathbb{Q}\left(\zeta_{n}\right)$ be a quadratic field of discriminant $\Delta_{d}$. Then there exists $\sigma \in \operatorname{Gal}\left(\mathbb{Q}\left(\zeta_{n}\right) / \mathbb{Q}\right)$ such that $\left(\left.\sigma\right|_{\mathbb{Q}\left(\zeta_{l}\right)}\right) \neq \operatorname{id}_{\mathbb{Q}\left(\zeta_{l}\right)}$ for every odd prime $l$ dividing $n$ and, moreover, $\sigma(\sqrt{d})=-\sqrt{d}$.

Proof. Let $\sigma_{a} \in \operatorname{Gal}\left(\mathbb{Q}\left(\zeta_{n}\right) / \mathbb{Q}\right)$ with $\sigma_{a}:=\zeta_{n}^{a}$ and $(a, n)=1$. It is well known that $\sigma(\sqrt{d})=\sqrt{d}$ if and only if $\left(\Delta_{d} / a\right)=1$, where $\left(\Delta_{d} / a\right)$ denotes the Kronecker symbol. Thus the problem reduces to showing that there exists $1 \leq a \leq n,(a, n)=1$ with $a \not \equiv 1(\bmod l)$ for every odd prime $l$ 
dividing $n$ and $\left(\Delta_{d} / a\right)=-1$. To prove that such an $a$ exists is left to the reader. (If $\Delta_{d}<0$, then $a=n-1$ is such an $a$.)

Proof of Theorem 5. We first prove the "if and only if" part of the assertion.

$\Leftarrow$. If $\sqrt{g} \in M$, then there does not exist a $\sigma$ such that $\left(\left.\sigma\right|_{M}\right)=\mathrm{id}_{M}$ and $\left(\left.\sigma\right|_{\mathbb{Q}\left(\zeta_{2}, \sqrt{g}\right)}\right) \neq \operatorname{id}_{\mathbb{Q}\left(\zeta_{2}, \sqrt{g}\right)}$, thus, by Theorem $6, \delta(M, g)=0$.

$\Rightarrow$. If $l \nmid h$ and $l$ is odd, then $\mathbb{Q}\left(g^{1 / l}\right)$ is not normal and hence $\mathbb{Q}\left(\zeta_{l}, g^{1 / l}\right) \nsubseteq$ $M\left(\zeta_{\pi}\right)$. If $l \mid h$, then $\mathbb{Q}\left(\zeta_{l}, g^{1 / l}\right)=\mathbb{Q}\left(\zeta_{l}\right) \subseteq M\left(\zeta_{\pi}\right)$. Thus the $l$ such that $\mathbb{Q}\left(\zeta_{l}, g^{1 / l}\right) \subseteq M\left(\zeta_{\pi}\right)$ are precisely the prime divisors of $\pi$ and possibly 2 . The (easier) case where 2 does not occur is left to the reader, so we may assume that $\sqrt{g} \in M\left(\zeta_{\pi}\right)$. Notice that we are done if we show that if $\sqrt{g} \notin$ $M$, then there exists $\sigma \in \operatorname{Gal}\left(M\left(\zeta_{\pi}\right) / \mathbb{Q}\right)$ such that $\sigma(\sqrt{g})=-\sqrt{g}$ and $\left(\left.\sigma\right|_{\mathbb{Q}\left(\zeta_{l}\right)}\right) \neq \mathrm{id}_{\mathbb{Q}\left(\zeta_{l}\right)}$ for every prime divisor $l$ of $\pi$.

Since by assumption $\sqrt{g} \in M\left(\zeta_{\pi}\right)$ and $M \subseteq \mathbb{Q}\left(\zeta_{f}\right), \sqrt{g} \in \mathbb{Q}\left(\zeta_{f}, \zeta_{\pi}\right)$. Put $(\pi, \Delta)^{*}=(-1)^{((\pi, \Delta)-1) / 2}(\pi, \Delta)$. As $\pi$ is odd, we see that $\sqrt{(\pi, \Delta)^{*}} \in \mathbb{Q}\left(\zeta_{\pi}\right)$ and, moreover, $\sqrt{(\pi, \Delta)^{*} \Delta} \in \mathbb{Q}\left(\zeta_{f}\right)$. We distinguish two cases:

(i) $\left[\mathbb{Q}\left(\sqrt{(\pi, \Delta)^{*}}\right): \mathbb{Q}\right]=2$. Let $\sigma_{1}=$ id $\in \operatorname{Gal}\left(\mathbb{Q}\left(\zeta_{f}\right) / \mathbb{Q}\right)$. Let $\sigma_{2}$ be an automorphism whose existence is asserted in Lemma 3 (with $n=\pi$ and $\left.d=(\pi, \Delta)^{*}\right)$. Since by assumption $(f, h)=1, \mathbb{Q}\left(\zeta_{f}\right)$ and $\mathbb{Q}\left(\zeta_{\pi}\right)$ are linearly disjoint and hence the automorphisms $\sigma_{1}$ and $\sigma_{2}$ can be lifted to an automorphism of $\mathbb{Q}\left(\zeta_{f}, \zeta_{\pi}\right)$. Take its restriction to $M\left(\zeta_{\pi}\right)$. This automorphism has all the required properties.

(ii) $\left[\mathbb{Q}\left(\sqrt{(\pi, \Delta)^{*}}\right): \mathbb{Q}\right]=1$. In this case $\sqrt{g} \in \mathbb{Q}\left(\zeta_{f}\right)$. Let $\sigma_{1} \neq$ id be the automorphism of $M(\sqrt{g})$ such that $\left(\left.\sigma_{1}\right|_{M}\right)=\left.\mathrm{id}\right|_{M}$. Since by assumption $\sqrt{g} \notin M, \sigma_{1}$ exists. Let $\sigma_{2} \in \operatorname{Gal}\left(\mathbb{Q}\left(\zeta_{\pi}\right) / \mathbb{Q}\right)$ be defined by $\sigma_{2}\left(\zeta_{\pi}\right)=\zeta_{\pi}^{-1}$. Since $M(\sqrt{g})$ and $\mathbb{Q}\left(\zeta_{\pi}\right)$ are linearly disjoint, $\sigma_{1}$ and $\sigma_{2}$ can be lifted to an automorphism of $\operatorname{Gal}\left(M\left(\zeta_{\pi}\right) / \mathbb{Q}\right)$. Notice that this automorphism has all the required properties.

The assertion regarding $N_{M}(g)$ is now easily deduced on using the latter part of Theorem 6 .

We demonstrate Theorem 5 by determining the set $\mathcal{L}$ of odd primes $l$ such that there are infinitely many primes $p$ satisfying $p \equiv \pm 1(\bmod l)$ with $l$ a primitive root $\bmod p$. Then we have to put $M=\mathbb{Q}\left(\zeta_{l}+\zeta_{l}^{-1}\right)$ and $g=l$ in Theorem 5. Since $\sqrt{l} \in \mathbb{R}$ and $M$ is the maximal real subfield of $\mathbb{Q}\left(\zeta_{l}\right)$, we find that $\sqrt{l} \in M$ if and only if $\sqrt{l} \in \mathbb{Q}\left(\zeta_{l}\right)$. Thus, using Lemma 1 , we see that on GRH, $\mathcal{L}=\{l: l \equiv 3(\bmod 4)\}$. Unconditionally it can be shown [8, Theorem 3.2] that $\mathcal{L}$ equals $\{l: l \equiv 3(\bmod 4)\}$ with at most two primes excluded. The fact that $\mathcal{L}$ is non-empty is used in A. Reznikov's [8] proof of a weaker version of a conjecture of Lubotzky and Shalev on three-manifolds. 
3. Proof of the main result. In this section Theorem 2 will be proved. First we carry out some preparations.

The next two lemmas are well known (cf. [3]).

Lemma 4. Let $M$ be a number field, $\kappa \in M$ and let $n \geq 1$ be an odd integer. If $\left[M\left(\zeta_{n}, \kappa^{1 / n}\right): M\right]=n \varphi(n)$, then $M\left(\zeta_{n}\right): M$ is the maximal abelian subextension of $M\left(\zeta_{n}, \kappa^{1 / n}\right): M$.

Proof. Let

$$
\mathcal{M}_{n}=\left\{\left(\begin{array}{ll}
1 & 0 \\
r & s
\end{array}\right): r \in \mathbb{Z} / n \mathbb{Z}, s \in(\mathbb{Z} / n \mathbb{Z})^{*}\right\} .
$$

One easily sees that commutators of $\mathcal{M}_{n}$ are of the form $\left(\begin{array}{ll}1 & 0 \\ \star & 1\end{array}\right)$. On noting that the commutator of $\left(\begin{array}{ll}1 & 0 \\ 0 & 2\end{array}\right)$ and $\left(\begin{array}{ll}1 & 0 \\ 1 & 1\end{array}\right)$ equals $\left(\begin{array}{ll}1 & 0 \\ 1 & 1\end{array}\right)$, it is seen that $\mathcal{M}_{n}^{\prime}$, the commutator subgroup of $\mathcal{M}_{n}$, equals $\left\{\left(\begin{array}{ll}1 & 0 \\ r & 1\end{array}\right): r \in \mathbb{Z} / n \mathbb{Z}\right\}$. It is enough to show that if the condition of the lemma is satisfied, then $\operatorname{Gal}\left(M\left(\zeta_{n}, \kappa^{1 / n}\right)\right.$ : $M) \cong \mathcal{M}_{n}$. For then the Galois group of the maximal abelian subextension of $M\left(\zeta_{n}, \kappa^{1 / n}\right): M$ is isomorphic to $\mathcal{M}_{n} / \mathcal{M}_{n}^{\prime} \cong(\mathbb{Z} / n \mathbb{Z})^{*}$. Since the maximal abelian subextension of $M\left(\zeta_{n}, \kappa^{1 / n}\right): M$ contains $M\left(\zeta_{n}\right): M$ and the condition of the lemma implies that the latter has Galois group $(\mathbb{Z} / n \mathbb{Z})^{*}$, we are done.

Let $\alpha$ be a root of $x^{n}-\kappa$. For any $\sigma \in \operatorname{Gal}\left(M\left(\zeta_{n}, \kappa^{1 / n}\right): M\right)$, there exist $l(\sigma) \in(\mathbb{Z} / n \mathbb{Z})$ and $m(\sigma) \in(\mathbb{Z} / n \mathbb{Z})^{*}$, such that $\sigma(\alpha)=\zeta_{n}^{l(\sigma)} \alpha$ and $\sigma\left(\zeta_{n}\right)=\zeta_{n}^{m(\sigma)}$. Now define a map $\psi \mapsto\left(\begin{array}{cc}1 & 0 \\ l(\sigma) & m(\sigma)\end{array}\right)$. One checks that it is a monomorphism of $\operatorname{Gal}\left(M\left(\zeta_{n}, \kappa^{1 / n}\right): M\right)$ into $\mathcal{M}_{n}$. Since $\left|\mathcal{M}_{n}\right|=n \varphi(n)$ and, by assumption, $\left|\operatorname{Gal}\left(M\left(\zeta_{n}, \kappa^{1 / n}\right): M\right)\right|=n \varphi(n), \psi$ is actually an isomorphism.

Lemma 5. Let $g \in G$ and $k$ be squarefree. Then the maximal abelian subextension of $\mathbb{Q}\left(\zeta_{k}, g^{1 / k}\right)$ is $\mathbb{Q}\left(\zeta_{k}\right)$ if $k$ is odd and $\mathbb{Q}\left(\zeta_{k}, \sqrt{g}\right)$ otherwise.

Proof. Write $g=\gamma_{1}^{h}, \gamma_{1} \in \mathbb{Q}$.

(i) $k$ is odd. By Lemmas 2 and $4, \mathbb{Q}\left(\zeta_{k}\right)$ is the maximal abelian subextension of $\mathbb{Q}\left(\zeta_{k}, \gamma_{1}^{1 / k}\right)$. Since $\mathbb{Q}\left(\zeta_{k}\right) \subseteq \mathbb{Q}\left(\zeta_{k}, g^{1 / k}\right) \subseteq \mathbb{Q}\left(\zeta_{k}, \gamma_{1}^{1 / k}\right)$, we are done in this case.

(ii) $k$ is even and $\sqrt{\gamma_{1}} \notin \mathbb{Q}\left(\zeta_{k}\right)$. Taking $M=\mathbb{Q}\left(\sqrt{\gamma_{1}}\right), \kappa=\sqrt{\gamma_{1}}$ and $n=k / 2$ in Lemma 4, we find, on using Lemma 2, that the maximal abelian subextension of $\mathbb{Q}\left(\zeta_{n}, \kappa^{1 / n}\right): \mathbb{Q}\left(\sqrt{\gamma_{1}}\right)$ equals $\mathbb{Q}\left(\zeta_{n}, \sqrt{\gamma_{1}}\right)=\mathbb{Q}\left(\zeta_{k}, \sqrt{g}\right)$. Since $\mathbb{Q}\left(\zeta_{k}, \sqrt{g}\right): \mathbb{Q}$ is abelian and

$$
\mathbb{Q}\left(\zeta_{k}, \sqrt{g}\right) \subseteq \mathbb{Q}\left(\zeta_{k}, g^{1 / k}\right) \subseteq \mathbb{Q}\left(\zeta_{k}, \gamma_{1}^{1 / k}\right)=\mathbb{Q}\left(\zeta_{n}, \kappa^{1 / n}\right),
$$

we are done. 
(iii) $k$ is even and $\sqrt{\gamma_{1}} \in \mathbb{Q}\left(\zeta_{k}\right)$. From Lemma 2 it follows that $\mathbb{Q}\left(\zeta_{k}, g^{1 / k}\right)$ $=\mathbb{Q}\left(\zeta_{k / 2}, g^{2 / k}\right)$. Since by assumption $4 \nmid k$, we are thus reduced to case (i).

Lemma 6. Let $g \in G$. If $g_{1} \equiv 1(\bmod 4)$ and $k$ is squarefree then, for $n \geq 0, \mathbb{Q}\left(\zeta_{k}, g^{1 / k}\right) \cap \mathbb{Q}\left(\zeta_{2^{n}}\right)=\mathbb{Q}$.

Proof. The intersection of the two fields under consideration must be abelian and is contained in $\mathbb{Q}\left(\zeta_{k}, \sqrt{g}\right)$ by Lemma 5 . Let $d_{K}$ denote the discriminant over $\mathbb{Q}$ of the number field $K$. Since the prime divisors of $d_{L_{1} \cdot L_{2}}$ all divide $d_{L_{1}} d_{L_{2}}$, we see that $d_{\mathbb{Q}\left(\zeta_{k}, \sqrt{g}\right)}$ is odd, on noting that $d_{\mathbb{Q}(\sqrt{g})}=g_{1}$, $d_{\mathbb{Q}\left(\zeta_{k}\right)}=d_{\mathbb{Q}\left(\zeta_{k / 2}\right)}$ for $k \equiv 2(\bmod 4)$ and that $d_{\mathbb{Q}\left(\zeta_{k}\right)}$ is not divisible by primes not dividing $k$. Thus 2 is not ramified at $\mathbb{Q}\left(\zeta_{k}, \sqrt{g}\right)$. On the other hand, every subfield of degree $>1$ of $\mathbb{Q}\left(\zeta_{2^{n}}\right)$ is ramified at 2 .

An integer is called $y$-smooth if all its prime divisors are $\leq y$.

Lemma 7. Let $d$ be 3 -smooth, but not 2-smooth. Let $g \in G$ be such that $g_{1}=21$ and $(h, 21)=7$. Let $k \geq 1$ be squarefree. Then $\mathbb{Q}\left(\zeta_{k}, g^{1 / k}\right) \cap \mathbb{Q}\left(\zeta_{d}\right) \subseteq$ $\mathbb{Q}(\sqrt{-3})$.

P r o o f. Using Lemma 5 it is seen that $\mathbb{Q}\left(\zeta_{k}, g^{1 / k}\right) \cap \mathbb{Q}\left(\zeta_{d}\right) \subseteq \mathbb{Q}\left(\zeta_{k}, \sqrt{21}\right)$ $\cap \mathbb{Q}\left(\zeta_{d}\right)$. Let $3^{\alpha} \| d$. Notice that $\mathbb{Q}\left(\zeta_{k}, \sqrt{g}\right)$ is not ramified at 2 (cf. the proof of the previous lemma). Thus $\mathbb{Q}\left(\zeta_{k}, \sqrt{21}\right) \cap \mathbb{Q}\left(\zeta_{d}\right) \subseteq \mathbb{Q}\left(\zeta_{k}, \sqrt{21}\right) \cap \mathbb{Q}\left(\zeta_{3^{\alpha}}\right)$. Now

$$
\mathbb{Q}\left(\zeta_{k}, \sqrt{21}\right) \cap \mathbb{Q}\left(\zeta_{3^{\alpha}}\right) \subseteq \mathbb{Q}\left(\zeta_{\mathrm{lcm}(k, 21)}\right) \cap \mathbb{Q}\left(\zeta_{3^{\alpha}}\right)=\mathbb{Q}\left(\zeta_{3}\right),
$$

where the latter equality follows on noticing that $\left(\operatorname{lcm}(k, 21), 3^{\alpha}\right)=3$.

REMARK. Actually under the conditions of Lemma 7 , we have $\mathbb{Q}\left(\zeta_{k}, g^{1 / k}\right)$ $\cap \mathbb{Q}\left(\zeta_{d}\right)=\mathbb{Q}(\sqrt{-3})$ if $3 \mid k$ or $14 \mid k$ and $\mathbb{Q}$ otherwise, but this will not be needed in the sequel.

Lemma 8. Let $g \in G$ and $l$ be an odd prime. Then $\delta\left(\mathbb{Q}\left(\zeta_{l}\right), g\right)=$ $\delta(\mathbb{Q}, g) / \varphi(l)$ if and only if $g$ is exceptional and $l=3$.

Corollary $1(\mathrm{GRH})$. Let $g \in G$ and $l$ be an odd prime. Then $\mathcal{P}_{g}$ is weakly uniformly distributed mod $l$ if and only if $g$ is exceptional and $l=3$.

Proof (of Lemma 8). Put $P(\alpha, \beta)=\prod_{p|\alpha, p| \beta}(p-2) \prod_{p \mid \alpha, p \nmid \beta}\left(p^{2}-p-1\right)$.

$\Leftarrow$. By Theorem 4 .

$\Rightarrow$. Notice that $l \nmid h$, for otherwise, by Theorem $4, \delta\left(\mathbb{Q}\left(\zeta_{l}\right), g\right)=0$, whereas $\delta(\mathbb{Q}, g)>0$. Notice also that $g_{1} \equiv 1(\bmod 4)$, for otherwise $\delta\left(\mathbb{Q}\left(\zeta_{l}\right), g\right)$ $=\delta(\mathbb{Q}, g) / \varphi(l)$ implies, by Theorem 4 , that $A(l, h)=A(1, h)$ and hence $1-(l-2) /\left(l^{2}-l-1\right)=1$, which is impossible. Then, since $g_{1} \equiv 1(\bmod 4)$, $l \nmid h$ and $\Delta=g_{1}$, the equality $\delta\left(\mathbb{Q}\left(\zeta_{l}\right), g\right)=\delta(\mathbb{Q}, g) / \varphi(l)$ implies, by Theorem 4 ,

$$
\left(1-\frac{\mu\left(\left|g_{1}\right|\right)}{P\left(g_{1}, h\right)}\right)=\left(1-\frac{l-2}{l^{2}-l-1}\right)\left(1-\frac{\mu(|b|)}{P(b, h)}\right)
$$


Now $l$ must divide $g_{1}$, for otherwise $b=g_{1}$ and hence $1-(l-2) /\left(l^{2}-l-1\right)=1$, which is impossible. Hence $b=g_{1} / l$ and thus (8) becomes

$$
\left(1-\frac{\mu\left(\left|g_{1}\right|\right)}{P\left(g_{1}, h\right)}\right)=\left(1-\frac{l-2}{l^{2}-l-1}\right)\left(1+\frac{\mu\left(\left|g_{1}\right|\right)\left(l^{2}-l-1\right)}{P\left(g_{1}, h\right)}\right) .
$$

Notice that $\mu\left(\left|g_{1}\right|\right)=1$. We find $P\left(g_{1}, h\right)=\left(l^{2}-l-1\right)\left(l^{2}-2 l+2\right) /(l-2)$. Since $\left(\left(l^{2}-l-1\right)\left(l^{2}-2 l+2\right), l-2\right)$ divides 2 and $P\left(g_{1}, h\right)$ must be an integer, it follows that $l=3$ and hence $P\left(g_{1}, h\right)=25$. Thus $g$ is exceptional and $l=3$.

Proof of Theorem 2. Assume that $g$ satisfies the assumptions of Theorem 2 and, moreover, assume GRH. Then by Theorem 4 with $f=1$ it follows that $\{1,2\} \subseteq D_{g}$. If $d \in D_{g}$ and $\delta$ divides $d$, then $\delta \in D_{g}$.

First consider the case where $g$ is ordinary. Then this observation together with Corollary 1 shows that $D_{g} \subseteq\left\{2^{n}: n \geq 0\right\}$. Suppose that $g_{1} \equiv 3$ (mod 4$)$. Then Theorem 4 shows that $\mathcal{P}_{g}$ is not weakly uniformly distributed $\bmod 4$. Thus in this case $D_{g}=\{1,2\}$. If $g_{1} \not \equiv 3(\bmod 4)$, then it is easy to see, by Theorem 4 again, that $4 \in D_{g}$. If $g_{1} \equiv 2(\bmod 4)$ then Theorem 4 again yields that $\mathcal{P}_{g}$ is not weakly uniformly distributed mod 8 . Thus in this case $D_{g}=\{1,2,4\}$. Finally assume that $g_{1} \equiv 1(\bmod 4)$. As we have seen, $D_{g} \subseteq\left\{2^{n}: n \geq 0\right\}$. Theorem 4 shows that $\delta\left(\mathbb{Q}\left(\zeta_{2^{n}}\right), g\right)=\delta(\mathbb{Q}, g) / \varphi\left(2^{n}\right)$. This is consistent with weak uniform distribution $\bmod 2^{n}$. In fact, using a result of Lenstra [4], we will show that $\mathcal{P}_{g}$ is weakly uniformly distributed $\bmod 2^{n}$ for every $n \geq 3$. This then completes the proof in the case where $g$ is ordinary.

Let $a$ and $d$ be coprime. The set of primes $p$ such that $p \equiv a(\bmod d)$, $p \nmid g$, and $g$ is a primitive root $\bmod p$, equals $M=M\left(\mathbb{Q}, \mathbb{Q}\left(\zeta_{d}\right), \sigma_{a},\langle g\rangle, 1\right)$, where we used Lenstra's notation. Here $\sigma_{a}$ denotes the automorphism of $\operatorname{Gal}\left(\mathbb{Q}\left(\zeta_{d}\right) / \mathbb{Q}\right)$ determined by $\sigma_{a}\left(\zeta_{d}\right)=\zeta_{d}^{a}$. Under GRH the natural density $\delta_{a}$, of the set $M$ is, by $[4,(2.15)]$, equal to

$$
\delta_{a}=\sum_{k=1}^{\infty} \frac{\mu(k) c_{a}(k)}{\left[\mathbb{Q}\left(\zeta_{d}, \zeta_{k}, g^{1 / k}\right): \mathbb{Q}\right]},
$$

where $c_{a}(k)=1$ if $\sigma_{a}$ fixes $\mathbb{Q}\left(\zeta_{k}, g^{1 / k}\right) \cap \mathbb{Q}\left(\zeta_{d}\right)$ pointwise and $c_{a}(k)=0$ otherwise. In case $g_{1} \equiv 1(\bmod 4)$ and $d=2^{n}$, by Lemma 6 the latter intersection of fields equals $\mathbb{Q}$ (at least when $k$ is squarefree) and hence $c_{a}(k)=1$ for every squarefree $k$. Thus $\delta_{a}=\delta_{1}$. This and $\delta_{1}=\delta\left(\mathbb{Q}\left(\zeta_{2^{n}}\right), g\right)>$ 0 , which follows by Theorem 4 (or alternatively Theorem 5), yield that $\mathcal{P}_{g}$ is weakly uniformly distributed $\bmod 2^{n}$.

It remains to deal with the case where $g$ is exceptional. By Corollary 1 , a necessary condition for $\mathcal{P}_{g}$ to be weakly uniformly distributed mod $d$ is that 
$d$ is 3 -smooth. The proof of the theorem will be completed once we show that this condition is also sufficient. The analysis of the case $g_{1} \equiv 1(\bmod 4)$ applies in the exceptional case as well and we find that for every 2 -smooth integer $d, \mathcal{P}_{g}$ is weakly uniformly distributed $\bmod d$. Next assume that $d$ is 3 -smooth, but not 2 -smooth. Let $a$ be an integer such that $(a, 6)=1$. By Lemma 7 it follows that $\mathbb{Q}\left(\zeta_{k}, g^{1 / k}\right) \cap \mathbb{Q}\left(\zeta_{d}\right) \subseteq \mathbb{Q}(\sqrt{-3})$ for squarefree $k$. Thus, by (9), there exist $\widetilde{\delta}_{1}$ and $\widetilde{\delta}_{-1}$ such that $\delta_{a}=\widetilde{\delta}_{1}$ if $\sigma_{a}$ fixes $\mathbb{Q}(\sqrt{-3})$ (that is, if $a \equiv 1(\bmod 3))$ and $\delta_{a}=\widetilde{\delta}_{-1}$ otherwise. Since, by Corollary 1 , $\mathcal{P}_{g}$ is weakly uniformly distributed $\bmod 3$, we see that

$$
\sum_{\substack{1 \leq a \leq d,(a, d)=1 \\ a \equiv 1(\bmod 3)}} \delta_{a}=\sum_{\substack{1 \leq a \leq d,(a, d)=1 \\ a \equiv-1(\bmod 3)}} \delta_{a},
$$

that is, $\varphi(d) \widetilde{\delta}_{1} / 2=\varphi(d) \widetilde{\delta}_{-1} / 2$. Since $\delta_{1}>0$ (by Theorem 5 for example), it follows that $\mathcal{P}_{g}$ is weakly uniformly distributed $\bmod d$.

REMARK 1. In the exceptional case the only integers that can be shown to be in $D_{g}$ by appealing to Theorem 4 only, are 1,2,3,4,6 and 12 .

REMARK 2. It is instructive to try to apply the argument that showed that $\mathcal{P}_{g}$ is weakly uniformly distributed modulo 2 -smooth numbers in case $g_{1} \equiv 1(\bmod 4)$ to $g$ satisfying $g_{1} \not \equiv 1(\bmod 4)$. Then we already know that $\mathcal{P}_{g}$ is not weakly uniformly distributed mod $2^{n}$ for $n$ large enough. Thus $c_{a}(k) \neq 1$ for some $a$ and squarefree $k$, that is, Lemma 6 must be false in this case. Indeed, if $g_{1} \equiv 3(\bmod 4)$, then $\mathbb{Q}\left(\zeta_{2\left|g_{1}\right|}, g^{1 /\left(2\left|g_{1}\right|\right)}\right) \cap \mathbb{Q}\left(\zeta_{2^{n}}\right) \supseteq \mathbb{Q}(i)$ for $n \geq 2$. If $g_{1} \equiv 2(\bmod 4)$ then, for $n \geq 3, \mathbb{Q}\left(\zeta_{\left|g_{1}\right|}, g^{1 /\left|g_{1}\right|}\right) \cap \mathbb{Q}\left(\zeta_{2^{n}}\right)$ contains $\mathbb{Q}(\sqrt{2})$ (respectively $\mathbb{Q}(\sqrt{-2})$ ) if $g_{1} / 2 \equiv 1(\bmod 4)$ (respectively $g_{1} / 2 \equiv 3$ $(\bmod 4))$.

The next lemma together with Theorem 2 immediately implies Theorem 3.

LemmA 9. Let $d \geq 1$ and $g \in G$. We have $\mathbb{Q}\left(\zeta_{k}, g^{1 / k}\right) \cap \mathbb{Q}\left(\zeta_{d}\right)=\mathbb{Q}$ for every squarefree $k$ if and only if (i), (ii) or (iii) of Theorem 2 is satisfied.

Proof. $\Rightarrow$. Suppose $d$ contains an odd prime factor, $p$. Then $\mathbb{Q}\left(\zeta_{p}\right) \subseteq$ $\mathbb{Q}\left(\zeta_{p}, g^{1 / p}\right) \cap \mathbb{Q}\left(\zeta_{d}\right)$ and thus $d=2^{n}$ for some $n \geq 0$. Suppose that $g_{1} \equiv 2(\bmod 4)$. We have to show that $n \leq 2$. So assume that $n \geq 3$. Then $\mathbb{Q}\left(\zeta_{\left|g_{1}\right|}, g^{1 /\left|g_{1}\right|}\right) \cap \mathbb{Q}\left(\zeta_{2^{n}}\right)$ contains $\mathbb{Q}(\sqrt{2})$ (respectively $\mathbb{Q}(\sqrt{-2})$ ) if $g_{1} / 2 \equiv 1(\bmod 4)\left(\right.$ respectively $\left.g_{1} / 2 \equiv 3(\bmod 4)\right)$. Finally suppose that $g_{1} \equiv 3(\bmod 4)$. We have to show that $n \leq 1$. So assume that $n \geq 2$. Notice that then $\mathbb{Q}(i) \subseteq \mathbb{Q}\left(\zeta_{2\left|g_{1}\right|}, g^{1 / 2\left|g_{1}\right|}\right) \cap \mathbb{Q}\left(\zeta_{2^{n}}\right)$. 
$\Leftarrow$. If $g_{1} \equiv 1(\bmod 4)$, then this follows by Lemma 6 . The other cases, except $g_{1} \equiv 2(\bmod 4)$ and $d=4$, are trivial. It remains to show that $i \notin \mathbb{Q}\left(\zeta_{k}, g^{1 / k}\right)$ for $k$ squarefree and $g_{1} \equiv 2(\bmod 4)$. A way of showing that $i \notin \mathbb{Q}\left(\zeta_{k}, g^{1 / k}\right)$ is to show that $\left[\mathbb{Q}\left(\zeta_{\operatorname{lcm}(4, k)}, g^{1 / k}\right): \mathbb{Q}\right]=2\left[\mathbb{Q}\left(\zeta_{k}, g^{1 / k}\right): \mathbb{Q}\right]$. This now follows by computing these degrees using Lemma 2 .

4. Conclusion. Let $g \in G$ and assume GRH. We have seen that to a large extent the equidistribution of the primes of $\mathcal{P}_{g}$ over the residue classes mod $d$ can be understood already from knowing whether or not the progression $1(\bmod d)$ gets its fair share of primes from $\mathcal{P}_{g}$. From Lemma 8 and Corollary 1, one sees that in case $d$ is an odd prime it is even true that the progression $1(\bmod d)$ gets its fair share if and only if all primitive progressions get their fair share. A question that thus naturally arises is whether this holds true for arbitrary $d$ (if so this would be rather surprising). Despite a considerable computational effort (together with Karim Belabas), I was not able to find a $d$ for which this is false. On the other hand, I obtained only partial non-existence results for such $d$.

The author thanks K. Belabas, T. Kleinjung, F. Lemmermeyer, A. Schinzel and P. Stevenhagen for helpful (e-mail) discussions and the referee for his comments (which led to a shortening of some of the proofs). This research was carried out at the Max-Planck-Institut in Bonn, the pleasant research atmosphere of which is gratefully acknowledged.

\section{References}

[1] R. Heath-Brown, A remark on Artin's conjecture, Quart. J. Math. Oxford Ser. (2) 37 (1986), 27-38.

[2] C. Hooley, Artin's conjecture for primitive roots, J. Reine Angew. Math. 225 (1967), 209-220.

[3] E. T. Jacobson and W. Y. Vélez, The Galois group of a radical extension of the rationals, Manuscripta Math. 67 (1990), 271-284.

[4] H. W. Lenstra, Jr., On Artin's conjecture and Euclid's algorithm in global fields, Invent. Math. 42 (1977), 201-224.

[5] —, Perfect arithmetic codes, Sém. Delange-Pisot-Poitou, 19e année 1978/79, Théorie des nombres, Fasc. 1, Exp. 15, 14 pp.

[6] P. Moree, On a conjecture of Rodier on primitive roots, Abh. Math. Sem. Univ. Hamburg 67 (1997), 165-171.

[7] W. Narkiewicz, Uniform Distribution of Sequences of Integers in Residue Classes, Lecture Notes in Math. 1087, Springer, 1984.

[8] A. Reznikov and P. Moree, Three-manifold subgroup growth, homology of coverings and simplicial volume, Asian J. Math. 1 (1997), 764-768. 
[9] F. Rodier, Estimation asymptotique de la distance minimale du dual des codes BCH et polynômes de Dickson, Discrete Math. 149 (1996), 205-221.

[10] E. Weiss, Algebraic Number Theory, New York Univ. Press, New York, 1963.

Max-Planck-Institut für Mathematik

Gottfried-Claren-Straße 26

53225 Bonn, Germany

E-mail: moree@mpim-bonn.mpg.de
Present address:

Faculteit WINS

Universiteit van Amsterdam Plantage Muidergracht 24

1018 TV Amsterdam, The Netherlands

E-mail: moree@wins.uva.nl

Received on 7.4 .1997

and in revised form on 3.12.1998 